\title{
A low voltage bootstrapped switch based on zero DC offset input voltage
}

\author{
Alihosein Sepahvand ${ }^{1}$ and Omid Hashemipour ${ }^{2 a)}$ \\ ${ }^{1}$ Microelctronic Lab, Shahid Beheshti University, G. C. Tehran, Iran \\ ${ }^{2}$ Department of Electrical Engineering, Shahid Beheshti University, G. C. Tehran, \\ Iran \\ a)hashemipour@sbu.ac.ir
}

\begin{abstract}
This paper presents an enhancement to the bootstrapped switching circuit by improving the input range limitation for applying direct input with no dc offset voltage. Application of a Darlington pair in the structure of the conventional bootstrap circuit allows for proper operation of circuits requiring low supply voltage and direct high input swing signal with no dc offset voltage. The proposed circuit is simulated using HSPICE and $0.18 \mu \mathrm{m}$ CMOS technology. Total Harmonic Distortion (THD) measurements results in SNR values which meet the necessary performance requirements for low-voltage application of switched capacitor circuits.
\end{abstract}

Keywords: bootstrapped switch, high input range, direct input, track and hold

Classification: Integrated circuits

\section{References}

[1] A. M. Abo and P. R. Gray, "A 1.5-V 10-bit 14.3-MS/s CMOS Pipeline Analog-to-Digital Converter," IEEE J. Solid-State Circuits, vol. 34, pp. 599-606, May 1999.

[2] T. S. Lee and C. C. Lu, "A 1.5 V 50-MHz pseudo-differential sample-andhold circuit with low hold pedestal," IEEE Trans. Circuit Syst. I, vol. 52, pp. 1752-1757, Sept. 2005.

[3] C. Lillebrekke, C. Wulff, and T. Ytterdal, "Bootstrapped switch in low voltage digital $90 \mathrm{~nm}$ CMOS technology," Proc. NORCHIP Conf., pp. 234236, Nov. 2005.

[4] G. W. Roberts "Calculating distortion levels in sampled-data circuits using Spice," Proc. IEEE International symposium on Circuits Syst., vol. 3, pp. 2059-2062, May 1995.

\section{Introduction}

Design of analog integrated circuit under low voltage condition is becoming more and more complicated. In case of analog switches this problem is also observed. Bootstrapping $[1,2,3]$ seems to be a better solution for low voltage 
switching of MOS transistors. However the operation of the bootstrapped switches depend on the input dc offset voltage and without this offset voltage the high input swing applications are not possible.

In this work, a Darlington pair configuration is utilized in order to apply direct high swing input voltage with no offset voltage. Experimental results based on a simple track and hold circuit shows the low distortion output under the above condition.

\section{Proposed Bootstrapped Switch}

Fig. 1 shows the transistor implementation of the proposed circuit. During the tracking phase $\left(\varphi_{1}\right)$ Darlington pair transistors M7-M8 and transistor M9 are switched off and the circuit operates in the same manner as previously explained by [2]. In holding phase $\left(\varphi_{2}\right)$, the main transistor switch, M11 must be turned off regardless of the input voltage value. The state of transistors M7-M9 in $\varphi_{2}$ depends on the input voltage level and this is where the main difference with conventional bootstrapped switch is observed.

Under hold condition $\varphi 2$, the circuit operates under two input conditions. When VIN $>0$ then, transistor M9 through VDD- $\mathrm{V}_{\mathrm{GS} 10}$, runs to triode region and because transistor M10 is always on and $\mathrm{V}_{\mathrm{DS} 10}$ is almost zero, the gate voltage of M11 is set to a voltage less than $\mathrm{V}_{\text {th11 }}$. Under this condition, transistor $\mathrm{M} 7$ is off since $\mathrm{V}_{\mathrm{GS}}$ is less than $\mathrm{V}_{\text {th7 }}$ so transistors M7-M8 have no effect on the switch operation. However, when $V_{\text {in }}<0$ then transistor M7 turns on and sets the gate voltage of M11 close to $V_{\text {in }}$ hence turning transistor $\mathrm{M}_{11}$ off.

In previous bootstrapped circuits, during holding period, the gate voltage of the main switch is connected to the ground and lowering the input voltage below $-\left|V_{\text {th }}\right|$ results in turning on the main switch. In this work, the above problem is solved by the action of the Darlington pair transistors M7 and M8.

Reducing the input voltage results in an increase in $\mathrm{V}_{\mathrm{gs}}$ of $\mathrm{M} 7$. If $\mathrm{V}_{\mathrm{IN}}$ reduces below $-2\left|V_{\text {th }}\right|$ then $V_{\text {gs7 }}$ which is almost equal to $V_{d s 7}$ will be greater than $V_{\text {th11 }}$ and therefore the circuit will no longer hold the input signal.

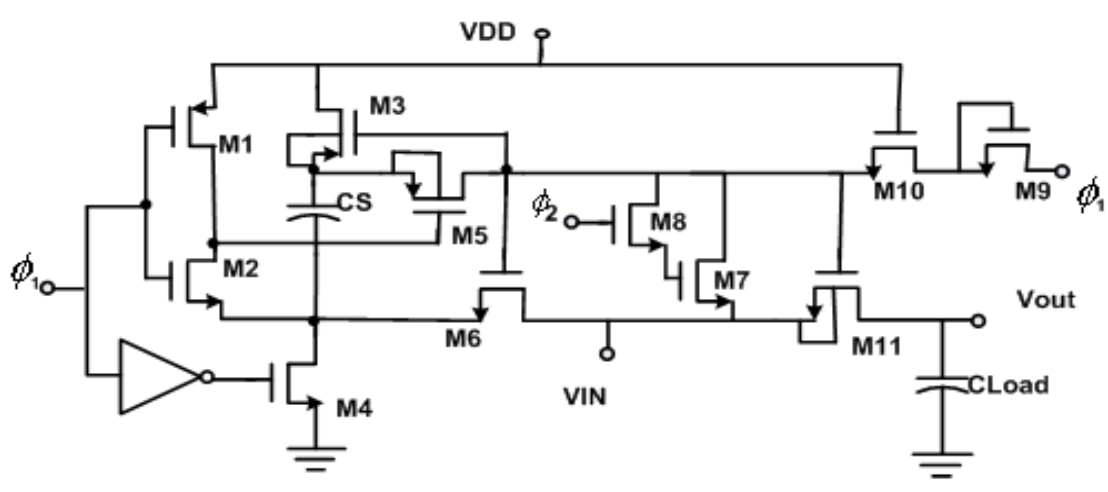

Fig. 1. Implementation of the proposed bootstrapped switch 


\section{Simulation Results}

The proposed bootstrapped switch is simulated using Hspice and $0.18 \mu \mathrm{m}$ CMOS technology. The supply voltage of $1.5 \mathrm{~V}$ and a sampling frequency of $100 \mathrm{MHz}$ with amplitude of $1.5 \mathrm{~V}$ are applied. Conventional [2] and proposed bootstrapped switch are applied to an elementary track and hold circuit utilizing simple NMOS switch with a capacitive load of 1.0 pF. Fig. 2 compares the transient responses with an input signal frequency of $3515625 \mathrm{~Hz}$ and amplitude of $2 \mathrm{~V}_{\mathrm{P}-\mathrm{P}}$ with no dc offset voltage.
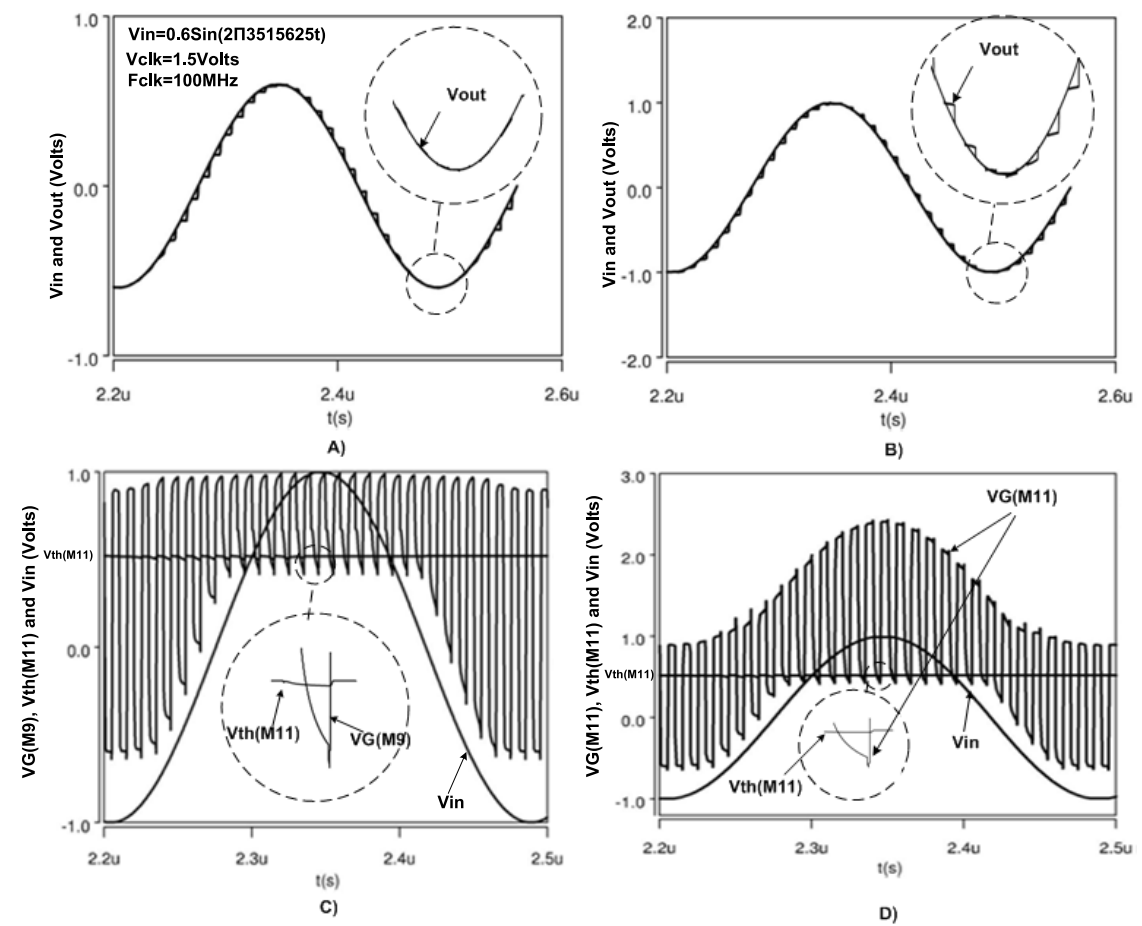

Fig. 2. Transient analysis A) Conventional bootstrapped switch B) Proposed bootstrapped switch C) Variation of the gate voltage of M9 versus $V_{\text {IN }} \mathrm{D}$ ) Variation of the gate voltage of M11versus $\mathrm{V}_{\text {IN }}$

As shown in Fig. 2 (a) the conventional circuit fails to hold the input signal during the holding period while input signal is lower than $-\left|V_{\text {th }}\right|$. Fig. $2(b)$ illustrates the proper operation of the proposed circuit for full swing input. As depicted in Fig. 2 (c) and Fig. 2 (d) in hold period the gate voltage of M9 that is almost equal to the gate voltage of M11 sets to a voltage lower than $\mathrm{V}_{\text {th11 }}$ and so turns M11 off. Fig. 3 is the spectrum analysis of the output signal using 4096-point FFT method [4] and input frequency of $3515625 \mathrm{~Hz}$. As illustrated in Fig. 3 the second and third harmonic distortion values are -79 and $-88 \mathrm{~dB}$ respectively. The SNR value of the proposed bootstrapped switch for the simple track and hold circuit is calculated to be $91 \mathrm{~dB}$.

\section{Conclusion}

In this work the shortcoming of a conventional bootstrapped circuit for appli- 


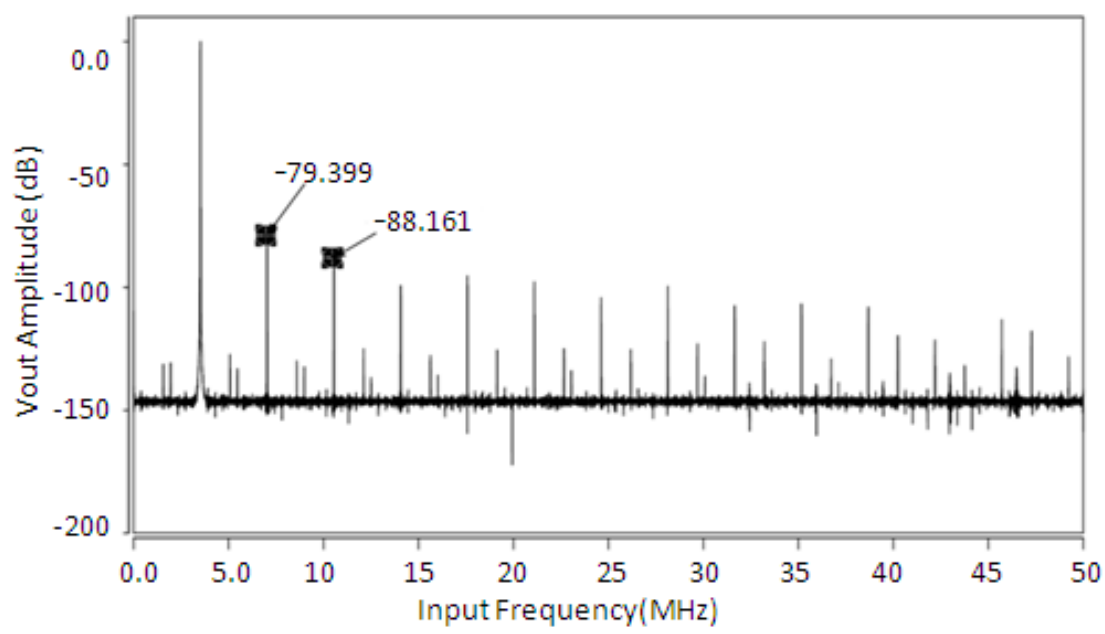

Fig. 3. The spectrum of the proposed circuit

cation of direct full swing input is solved using the behavior of a Darlington pair combination. Simulation results using a simple track and hold circuit and a capacitive load of $1 \mathrm{pF}$ shows a SNR value of $91 \mathrm{~dB}$. Simulations are carried out with Hspice and $0.18 \mu \mathrm{m}$ CMOS technology. 